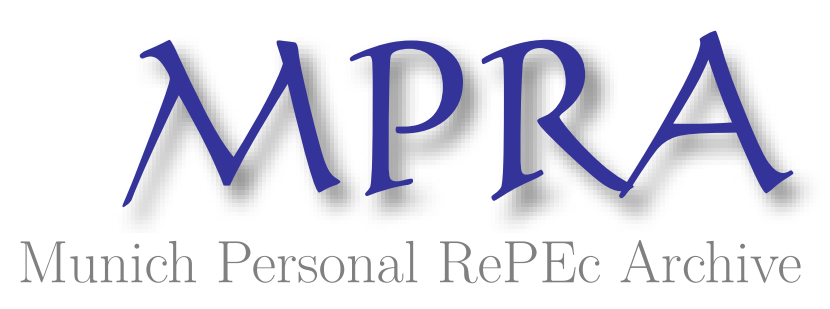

\title{
Choice of financing mode as a stochastic bounded control problem
}

\author{
Miglo, Anton
}

2014

Online at https://mpra.ub.uni-muenchen.de/56323/

MPRA Paper No. 56323, posted 06 Jun 2014 09:18 UTC 


\title{
Choice of financing mode as a stochastic bounded control problem
}

\author{
Anton Miglo*
}

2014

Key words: optimal financing, stochastic optimization, bounded control, asymmetric information, debt.

${ }^{*}$ Author affiliation: University of Bridgeport, 126 Park Ave., Bridgeport, CT 06604. Acknowledgments. I would like to thank Claude Fluet and the seminar participants at UQAM for the helpful discussions and comments. I am also thankful for the financial support awarded by the Montreal Institute of Financial Mathematics $\left(\mathrm{IFM}^{2}\right)$. 
Abstract. In this note I analyze situations where an entrepreneur needs external financing from an outside investor in order to start an investment project that will yield a profit for two consecutive periods. The value of second-period profit is the entreprneur's private information. I show that the choice of financing mode can be transformed into an optimal stochastic bounded control problem, where the state variable $t$ represents the investor's first-period payoff and the control variable $\alpha$ can be interpreted in terms of the investor's residual profit rights. I then show that under certain general conditions such as the monotonicity and continuity of $t$ (which have clear economic interpretations), an optimal contract is characterized by maximal $\alpha$ under low values of $t$ and minimal $\alpha$ under high values of $t$. In economic terms this corresponds to debt.

\section{Introduction}

In this note I analyze situations in which a wealth-constrained entrepreneur needs financing from an outside investor in order to start an investment project. In their famous proposition Modigliani and Miller (1958) established that when markets are perfect, the choice of financing mode plays no role. In practice however, the irrelevance of financial decisions is not observed. One of the most intriguing challenges in this field has been to explain why debt contracting and accordingly debt-like securities such as deposit certificates, bonds, bills of exchange, etc. play such important roles in financing.

I assume that the entrepreneur possesses private information regarding the firm's profit. Nachman and Noe (1994) resolved the problem of optimal financing in a one-period environment with asymmetric information. My analysis involves a two-period model and, in contrast to existing literature, there is asymmetry between the entrepreneur and the investor in terms of future profit, while the distribution of first-period profit is common to both.

When the profit generated by the project is a continuous variable, the choice of financing can be described as a stochastic optimization problem, wherein the unknown function represents the payoff to the investor. Furthermore, I show that the entrepreneur's problem is an optimal bounded control problem and that it is equivalent to minimizing the expected value of the control variable $\alpha$ for a given average value of $t$, where $t$ represents the first-period payoff to the investor and $\alpha$ can be interpreted in terms of its 
second-period profit rights. The reason for minimizing $\alpha$ stems from the fact that the asymmetric information pertains to the second period, and thus by minimizing the investor's residual rights the entrepreneur can mitigate the adverse selection problem. This problem can in turn be transformed into a stochastic bounded control problem with an isoparametric constraint (see Kamien and Schwartz, 1981). Consequently I show that under certain general conditions such as monotonicity and continuity of $t$ (which have clear economic interpretations), an optimal contract would have maximal $\alpha$ under low values of $t$ and minimal $\alpha$ under high values of $t$. In economic terms this corresponds to debt.

The rest of the paper is organized as follows: Section 2 describes the model; Section 3 shows results obtained regarding the optimality of debt; Section 4 discusses assumptions made by this paper and Section 5 contains the conclusions.

\section{Model.}

Consider a firm that has to make an investment $b>0$. There is no cash or assets in place available and therefore the firm needs external financing from an outside investor. The firm's owner (the entrepreneur) and the investor are risk neutral. In period 1, the firm's cash flow is $r \in[0, \bar{r}]$ with the distribution function $F(r)$ and density function $f(r)$, which are common knowledge. Except the cash, after the liquidation there are some assets which represent specific investments and which can not be sold immediately after the liquidation. ${ }^{1}$ However they yield the revenue $c$ in the second period. $c$ can either be $c_{h}$ with probability $p$ or $c_{l}$ with probability $1-p, c_{h}>c_{l}>0 . p$ is common knowledge. Before issuing securities and making investments, the entrepreneur receives private information about the value of $c$, which is not available to the investor. All available cash is distributed to the claimholders according to issued securities immediately after the liquidation. At the same time the parties should establish the property rights on the remaining assets (RR).

First-period profit does not cover the cost of investment, while the total project's NPV is positive for each firm, i. e.

$$
E r<b<E r+c_{j}, j=l, h
$$

\footnotetext{
${ }^{1}$ See Hart (1995) about the relationship-specific investments.
} 
where $E$ denotes mathematical expectation. From (1) the parties cannot write short-term (one-period) financing contract in order to avoid asymmetric information problems in the second period.

The investor's contract is $[t(r), \alpha(r)]$, where $t(r)$ is his first-period payoff, $\alpha(r)$ is his RR (accordingly $\alpha(r) c$ is his residual payoff). From a practical point of view, the property rights $\alpha(r)$ should be established immediately after liquidation and thus cannot be contingent on $c$. The typical argument in the literature is that complete contracts determining the residual payoffs in any state of nature are impossible to write. ${ }^{2}$ This is especially true for after-liquidation activities which are very difficult to describe ex-ante. Hence the residual owner(s) should be determined. Since all available cash is immediately distributed after liquidation, we can write:

$$
\begin{aligned}
t_{E}(r) & =r-t(r), \forall r \\
\alpha_{E}(r) & =1-\alpha(r), \forall r
\end{aligned}
$$

where $t_{E}(r)$ is the entrepreneur's first-period payoff and $\alpha_{E}(r)$ is his RR.

Let us assume:

(i) $t(r)$ and $t_{E}(r)$ are monotone increasing functions from $[0, \bar{r}]$ into $[0, \bar{r}]$. (ii)

$$
\alpha(r)=t^{\prime}(r)
$$

if $t^{\prime}(r)$ exists.

Most known securities correspond to this rule (see Section 4 for a discussion).

Note that assumptions (i) and (ii) imply that

$$
0 \leq \alpha(r) \leq 1, \forall r
$$

if $t^{\prime}(r)$ exists. This in turn together with assumption (i) implies limited liability for the parties (the payoffs are non-negative) in both periods (see Innes (1990) for a discussion).

(iii) The distribution function $F$ follows "increasing hazard-rate condition" (IHRC), i. e. $f /(1-F)$ is increasing in $r$.

Given (2), (3) and (4), the security design can be fully described by the investor's first-period payoff $t(r)$. Let the game be as follows:

1. The type of the entrepreneur ( $h$ or $l$ ) is revealed.

\footnotetext{
${ }^{2}$ See, for example, Hart (1995).
} 
2. He chooses a financial contract $t(r)$ and proposes this contract to the investor in exchange for an amount $b$.

3. The investor accepts or rejects the offer. If the investor rejects the offer, the entrepreneur's payoff equals zero. If the investor accepts, the entrepreneur issues the securities contracted above and invests $b$.

4. First-period profit $r$ is realized. The investor's first-period payoff is $t(r)$ and that of the entrepreneur is $r-t(r)$. RR become known: $t^{\prime}(r)$ for the investor and $1-t^{\prime}(r)$ for the entrepreneur.

5. Second-period benefit $c$ is realized. The investor's second-period payoff is $t^{\prime}(r) c$ and the entrepreneur's $\left(1-t^{\prime}(r)\right) c$.

The entrepreneur maximizes his expected payoff. Since there are no assets in place available and no other opportunities for the entrepreneur, limited liability implies that the entrepreneur will always do better by investing. The investor will accept the contract if his expected payoff is at least $b$. Throughout this article, I use the concept of Perfect-Bayesian equilibria and also verify that off-equilibrium beliefs survive usual refinements like Cho and Kreps' (1987) intuitive criterion. If several equilibria exist then the solution is determined by minimizing the mispricing for undervaluing type. ${ }^{3}$ Which contract is optimal for the entrepreneur?

If information about the firm's type is symmetric, then the optimal contract for the entrepreneur of type $j$ can be given by any contract $t(r)$ such that the investor's budget constraint is binding in equilibrium: $E\left[t(r)+t^{\prime}(r) c_{j}\right]=$ $b$. The entrepreneur's payoff will in this case be: $E\left[r-t(r)+\left(1-t^{\prime}(r)\right) c_{j}\right]=$ $E r+c_{j}-b$ (first-best output).

\section{The solution of the game with asymmetric information.}

The outline of the solution is as follows. Intuitively, the solution is pooling equilibrium, given that a low-profit type $l$ mimic always a high-profit type $h$. Which pooling minimizes mispricing for $h$ ? The basis of mispricing is asymmetric information relative to further profit. The idea is that the optimal contract should be one that minimizes the average investor's $R R$ and maximizes his average first-period revenue. In this case mispricing would be minimal. Another point is that debt is the contract that provides maximal

\footnotetext{
${ }^{3}$ See, for instance, Nachman and Noe (1994).
} 
slope under low values of $r$. Given the continuity of the contracts this allows to reach high payments for relatively low values of $r$ comparatively to other contracts and support these high payments without increasing slope for high values of $r$. This minimizes the average slope of the investor's contract.

Let $W_{j}(t)$ represent the type $j$ entrepreneur's expected total payoff given the investor's contract $t$ (if accepted) and $W(t, \mu)$ be the investor's expected payoff given the contract $t$ and the probability $\mu$ that the firm is type $h$. Obviously:

$$
\begin{gathered}
W_{j}(t)=E\left(r-t(r)+\left(1-t^{\prime}(r) c_{i}\right)\right. \\
W(t, \mu)=\mu\left(E t(r)+t^{\prime}(r) c_{h}\right)+(1-\mu)\left(E t(r)+t^{\prime}(r) c_{l}\right)
\end{gathered}
$$

From (6) and (7) we get

$$
W_{h}(t)=E r+c_{h}-W(t, 1)
$$

and

$$
W_{l}(t)=E r+c_{l}-W(t, 0)
$$

The equations (8) and (9) correspond to the case of symmetric information.

Lemma 1. $W(t, \mu)$ is increasing in $\mu$.

Proof. Follows immediately from (7) and $c_{h}>c_{l}$. End proof.

This lemma has two following important implications.

Lemma 2. The investor would accept (on equilibrium path or out-of) any contract $t$ such as $W(t, 0)=b$.

Proof. According to lemma 1, the investor's payoff observing the contract $t$ is minimized if the type is $l$ (which corresponds to the case $\mu=0$ ). If in this case the investor's payoff is $b$, the investor will accept. End proof.

Lemma 3. The type $l$ is never undervalued in equilibrium.

Proof. The proof is by contradiction. Suppose that $l$ is undervalued in equilibrium or that $W_{l}(t)<E r+c_{l}-b$. Then $l$ would deviate in any contract described in lemma 2. Since this contract will be accepted, we get from (9) that the payoff of $l$ would be $E r+c_{l}-b$. End proof. 
The following exercise is crucial in this article. Suppose that the investor accept any contract $t$ such that $W(t, \mu) \geq b$ for some given $\mu$. What is the best contract for type $h$ ?

Lemma 4. Consider the following optimization problem: $\max _{t(r)} W_{h}(t)$ under the constraint: $W(t, \mu) \geq b$. The solution to this problem is debt.

Proof. By definition of debt, we should show that the solution has the form $t(r)=\min \left\{r, r^{*}\right\}$ for some given $r^{*}{ }^{4}$ First, $t$ is continuous and differentiable almost everywhere and

$$
\begin{gathered}
t(0)=0 \\
0 \leq t^{\prime}(\cdot) \leq 1
\end{gathered}
$$

For continuity see Harris and Raviv (1988, lemma 1). If a monotone function is continuous, it must be differentiable almost everywhere. ${ }^{5}$ The condition (10) follows from assumption (i) considering $r=0$. The condition (11) follows from the assumption (ii) and the condition (5). Now present this problem in the form of an optimal control problem. This is:

$$
\max _{t(\cdot)} \int_{0}^{\bar{r}}\left[r-t(r)+c_{h}\left(1-t^{\prime}(r)\right)\right] f(r) d r
$$

subject to:

$$
\begin{gathered}
\mu E\left[t(r)+t^{\prime}(r) c_{h}\right]+(1-\mu) E\left[t(r)+t^{\prime}(r) c_{l}\right] \geq b \\
t(0)=0 \\
0 \leq t^{\prime}(r) \leq 1, \forall r
\end{gathered}
$$

Given the condition (14), this is an optimal bounded control program (see, for example, Kamien and Schwarz, 1981). We can simplify this problem to the following:

$$
\min _{t(\cdot)} \int_{0}^{\bar{r}}\left(t(r)+c_{h} t^{\prime}(r)\right) f(r) d r
$$

\footnotetext{
${ }^{4} r^{*}$ denotes debt face value.

${ }^{5}$ This is Lebesgue's theorem. See, for instance, Kolmogorov and Fomin (1998, p.321).
} 
subject to:

$$
\begin{gathered}
E t(r)+A E t^{\prime}(r) \geq b \\
t(0)=0 \\
0 \leq t^{\prime}(r) \leq 1, \forall r
\end{gathered}
$$

where $A=\mu c_{h}+(1-\mu) c_{l}$. The equation (15) is bounded in equilibrium. ${ }^{6}$ Thus, we can write

$$
E t(r)=b-A E t^{\prime}(r)
$$

We can further simplify the objective function to:

$$
\begin{gathered}
\min _{t(\cdot)}\left[b-A E t^{\prime}(r)+\int_{0}^{\bar{r}} c_{h} t^{\prime}(r) f(r) d r\right] \Leftrightarrow \\
\min _{t(\cdot)}\left[b+(1-\mu)\left(c_{h}-c_{l}\right) E t^{\prime}(r)\right]
\end{gathered}
$$

We can then rewrite the optimization problem as:

$$
\begin{gathered}
\min _{t(\cdot)} E t^{\prime}(r) \\
E t(r)+A E t^{\prime}(r)=b \\
t(0)=0 \\
0 \leq t^{\prime}(r) \leq 1, \forall r
\end{gathered}
$$

In order to solve this problem that contains isoparametric constraints (16), we will transform our problem into one with an end-point constraint. Inducing new variable $y: y^{\prime}(r)=t(r) f(r)+A t^{\prime}(r) f(r)$ allows us to transform the isoparametric constraints into an end-point constraint because the equation (16) is equivalent to $y(\bar{r})=b$. Our problem can be further transformed in this way:

$$
\min _{t(r), y(r), \alpha(r)} \int_{0}^{\bar{r}} \alpha(r) f(r) d r
$$

\footnotetext{
${ }^{6}$ Suppose that the contract $t(r)$ is optimal and (15) is not bounded. Let $R=\max (r$ $t(r)=0)$. Consider the contract $T(r)=0$ if $r \leq R+\varepsilon$ and $T(r)=(1-\varepsilon) t(r)$, if $r>R+\varepsilon$. It corresponds to the assumption (i) and betters the objective function because $T(r) \leq t(r)$ and $T^{\prime}(r) \leq t^{\prime}(r), \forall r$ where both inequlities are strict for some $r$ with positive mesure. It decreases the left side of (15). Therefore, by making $\varepsilon$ sufficiently small, the better contract is achieved.
} 


$$
\begin{gathered}
t(0)=0 \\
t^{\prime}=\alpha \\
0 \leq \alpha \leq 1 \\
y^{\prime}=t f+A \alpha f \\
y(0)=0 \\
y(\bar{r})=b
\end{gathered}
$$

Further, we can use standard optimization techniques. Hamiltonian is: $H=\alpha f+\lambda_{1} \alpha+\lambda_{2}[t f+A \alpha f]$ where $\lambda_{1}$ - is a co-state variable associated with (18) and, $\lambda_{2}$ - is co-state variable associated with (20). Associated Lagrangian is: $L=H-w_{1} \alpha-w_{2}(1-\alpha)$ where $w_{1}$ and $w_{2}$ are two multipliers associated with double-inequality (19). The optimal solution satisfies:

1. The Pontryagin principle with regard to control variable $\alpha$ :

$$
L_{\alpha}=f+\lambda_{1}+\lambda_{2} A f-w_{1}+w_{2}=0
$$

2. The Euler equations: 1) $\lambda_{2}^{\prime}=-\frac{\partial L}{\partial y} \Leftrightarrow$

$$
\lambda_{2}^{\prime}=0
$$

and 2) $\lambda_{1}^{\prime}=-\frac{\partial L}{\partial t} \Leftrightarrow$

$$
\lambda_{2} f=-\lambda_{1}^{\prime}
$$

3. The Kuhn-Tukker conditions:

$$
w_{1} \geq 0, w_{2} \geq 0, w_{1} \alpha=0, w_{2}(1-\alpha)=0
$$

4. The transversality condition (Kamien and Scwartz, 1981, p.148): 1) $H_{\alpha}^{\prime}(\bar{r})=0$ which implies:

$$
\lambda_{1}(\bar{r})=0
$$

These conditions allow us to solve the problem. The equation (24) implies:

$$
\lambda_{2}=D
$$

Substituting this in (25) and integrating it gives:

$$
D F(r)+K=-\lambda_{1}
$$


where $K$ is determined by using condition (27):

$$
K=-D
$$

Finally:

$$
\lambda_{1}=-D F(r)+D
$$

Thus: $H_{\alpha}(r) \equiv f+\lambda_{1}+\lambda_{2} A f=f(r)-D F(r)+D+D A f(r)$. The equations (23) and (26) imply:

$$
H_{\alpha}(r)<0 \Rightarrow w_{2}>0 \Rightarrow \alpha=1
$$

and:

$$
H_{\alpha}(r)>0 \Rightarrow w_{1}<0 \Rightarrow \alpha=0
$$

and:

$$
H_{\alpha}(r)=0 \Rightarrow w_{1}=w_{2}=0 \Rightarrow \alpha \in[0,1]
$$

Let us suppose that $1+D A<0$. This is only possible if $D<0$. But in this case, $H_{\alpha}(r)$ would be negative everywhere, which is impossible. Thus $1+D A>0$. In order to determine the sign of $H_{\alpha}$, the following inequality is crucial:

$$
\frac{f(r)}{1-F(r)}>,=,<-\frac{D}{1+D A}
$$

If $\frac{f(r)}{1-F(r)}>-\frac{D}{1+D A} \Longrightarrow \alpha=0$. From assumption (iii) $\frac{f(r)}{1-F(r)}$ is increasing. Thus if $\frac{D}{1+D A}>-f(0)$ then $\alpha=0$ everywhere which is impossible. Thus $\frac{D}{1+D A}<-f(0)$. In this case, $H_{\alpha}$ must change its sign only once. Let this point be $r^{*}$. We now have: $H_{\alpha}(r)<0, r \leq r^{*}$ and $H_{\alpha}(r) \geq 0, r>r^{*}$. This implies $\alpha=1, r \leq r^{*}$ and $\alpha=0, r>r^{*}$, making debt the solution. End proof.

Lemma 3 allows us to establish the following result about pooling with debt.

Proposition 1. Let $t_{d}$ be a debt contract such that:

$$
W\left(t_{d}, p\right)=b
$$

There exists a pooling equilibrium characterized by the following conditions: 1) both types play $t_{d}$; 2) off-equilibrium investor belief $\mu$ observing certain contracts $t$ is as follows: if

$$
W(t, 0)<W\left(t_{d}, 0\right)
$$


then $\mu=0$; if

$$
W(t, 0)>W\left(t_{d}, 0\right)
$$

then $\mu=1$; if

$$
W(t, 0)=W\left(t_{d}, 0\right)
$$

then any beliefs are possible.

If pooling equilibrium with $t \neq t_{d}$ exists when the mispricing under that is greater than under pooling with $t_{d}$.

Proof. Consider pooling with $t_{d}$. 1) $l$ does not deviate in another contract. The only incentives for $l$ to deviate to some $t$ occur when:

$$
W_{l}(t)>W_{l}\left(t_{d}\right)
$$

Given equ. (9) this is equivalent to:

$$
W(t, 0)<W\left(t_{d}, 0\right)
$$

However, all such contracts would not be accepted by the investor. The conditions (36), (37) and lemma 1 imply

$$
W(t, 0)<W\left(t_{d}, 0\right)<W\left(t_{d}, p\right)=b
$$

2) $h$ does not deviate. The proof is by contradiction. Suppose that $h$ deviates, meaning there exists $t_{1}(r)$ accepted by the investor such that:

$$
W\left(t_{1}, 1\right)<W\left(t_{d}, 1\right)
$$

Let us look at the following optimization problem:

$$
\min _{t(r)} W(t, 1)
$$

subject to:

$$
W(t, 0) \geq W\left(t_{d}, 0\right)
$$

We can apply lemma 4 . This is because given the equation (8) $\min _{t(r)} W(t, 1)$ is equivalent to $\max _{t(r)} W_{h}(t)$; in the condition (12) we can take $\mu=0$ and instead of $b$ to use $W\left(t_{d}, 0\right)$. The solution to this problem $t_{2}$ is a debt contract. Evidently the interest rate for this contract would not be less than it is for $t_{d}$ because the condition (41) implies $W\left(t_{2}, 0\right) \geq W\left(t_{d}, 0\right)$. This in turn implies $W\left(t_{2}, \mu\right) \geq W\left(t_{d}, \mu\right), \forall \mu$. Given (40) we can write:

$$
W\left(t_{2}, 1\right) \geq W\left(t_{d}, 1\right)>W\left(t_{1}, 1\right)
$$


This is a contradiction because if it is true, $t_{2}$ is not the solution for the described optimization problem. Since $t_{1}$ is accepted by the investor then, according to our system's description for off-equilibrium beliefs, we should have:

$$
W\left(t_{1}, 0\right) \geq W\left(t_{d}, 0\right)
$$

(if not then the contract is not accepted: see (39)) and thus $t_{1}(r)$ satisfies (41) and is better than $t_{2}(r)$; 3) Out-off equilibrium beliefs satisfy Cho and Kreps (1987) intuitive criterion. For the case (37) the investor belief that the type is $l$ is reasonable because the potential deviation of $l$ may be profitable for him. Now, in the case (38), the investor sets the probability zero on the type $l$ because he will never deviate to such a contract.

Let us analyze the last part of the proposition. Consider the following optimization problem:

$$
\max _{t(r)} W_{h}(t)
$$

subject to:

$$
W(t, p) \geq b
$$

The condition (36) and lemma 4 imply that $t_{d}$ is the solution to this problem (other debt contracts would have higher interest rates). Now suppose that there exists another pooling equilibrium, with $t_{a}$ being different from that described above and with smaller mispricing for $h$, i.e.

$$
W_{h}\left(t_{d}\right) \leq W_{h}\left(t_{a}\right)
$$

Obviously,

$$
W\left(t_{a}, p\right) \geq b
$$

But in this case, (42) contradict the fact that $t_{d}$ is the solution to the described above optimization problem. End Proof.

Proposition 1 establishes the existence of a pooling equilibrium with debt and describes completely this equilibrium. In particular, the investor's participation constraint is binding and off-equilibrium beliefs are such that if some contract gives the investor less than debt for type $l$, the investor is pessimistic about this contract and does not accept it. While if the payoff for some contract - even for a low-profitable type - is larger than in equilibrium, the investor believes that this is a high-profitable type and the investor accepts this contract. The condition (37) insures that very bad contracts will not be accepted and type $l$ will not deviate, while the condition (38) together 
with lemma 1 insures that type $h$ does not deviate. If it does deviate, bringing into existence some more efficient contract, this contract will also be debt because even the best contract among those satisfying (38) (which is debt according to lemma 4 but with different interest rates) cannot be better than $t_{d}$.

Finally consider separating equilibria.

Proposition 2. If separating equilibrium exists then the mispricing under that contract is greater than under pooling with $t_{d}$.

Proof. Consider any separating equilibrium and let $t_{h}$ and $t_{l}$ be the equilibrium contracts. Since equilibrium is separating we should have $W\left(t_{l}, 0\right) \geq$ $b$. From lemma 2 the investor accepts any contract with $W(t, 0)=b$. So in equilibrium it should be $W\left(t_{l}, 0\right)=b$ (if not $l$ will deviate). The incentive constraint for non-deviation of $l$ to the contract $t_{h}$ is $W_{l}\left(t_{h}\right)<E r+c_{l}-b$. Given (8) this is equivalent to

$$
W\left(t_{h}, 0\right)>b
$$

Consider the following optimization problem:

$$
\min _{t(r)} W(t, 1)
$$

subject to:

$$
W(t, 0) \geq b
$$

Here once again we can apply lemma 4 . This is because $\min _{t(r)} W(t, 1)$ is equivalent to $\max _{t(r)} W_{h}(t)$ given the equation (8); we can take $\mu=0$. The solution to this problem $t_{h}^{\prime}$ is a debt contract. First, note that $W_{h}\left(t_{h}^{\prime}\right) \geq$ $W_{h}\left(t_{h}\right)$. This is because $t_{h}$ satisfies (44): if not, $l$ will deviate to $t_{h}$. Second, the interest rate corresponding to $t_{h}^{\prime}$ is not less than for $t_{d}$. This follows from the fact that (44) is a stricter condition than (12). This follows from lemma 1. Finally: $W_{h}\left(t_{d}\right) \geq W_{h}\left(t_{h}^{\prime}\right) \geq W_{h}\left(t_{h}\right)$. End proof.

The result of this analyses is that pooling equilibrium with debt (with interest rates such that the investor's budget constraint is binding in equilibrium) is an equilibrium which survives the refinements criterion and which minimizes mispricing comparatively to other equilibria. 


\section{Discussion about assumptions.}

1. Increasing hazard-rate condition. An usual assumption in contract theory relative to distribution function is monotone likelihood ratio condition MLRP (see, for example, Jewitt, 1991). In the security design literature, some examples of using this condition can be found in Dewatripont and Tirole (1994), Innes (1990) and Matthews (2002). It can be shown that MLRP implies IHRC (see, for example, Wolfstatter, 1999). Thus, compared to MLRP, IHRC is weaker. Most of the known distribution functions satisfy IHRC. A well known example in financial contract literature is Nachman and Noe (1994).

2. Limited liability. For any type of enforceable contract, the absence of limited liability is not worth considering because no matter what the profit realization, payback is always possible and this leads to the possibility of first-best. Limited liability is the usual assumption made in the financial contract literature that discusses modern forms of business organization (such as public corporations etc.).

3. Monotonicity. The reasons for the use of the monotonicity condition were explained by Innes (1990) and Matthews (2002): if the contract is not monotonic then first, the entrepreneur can artificially increase profits by borrowing funds on the investor and second, investors are given incentives to destroy the firm's activities.

4. The rule of marginal revenues. By assumption (ii), if the currentperiod payoff is $t(r)$ then the residual cash-flow rights (residual property rights) should be $t^{\prime}(r)$. This is consistent with the standard securities. Take, for example, non-levered common equity. In this case both the entrepreneur's and the investor's contracts are linear: $t(r)=\alpha r$ and $t_{E}(r)=(1-\alpha) r$, where $\alpha$ shows the part of investor's shares in the total number of issued shares. His property rights (residual cash-flow rights) equals $\alpha \equiv t^{\prime}(r)$ which is obviously consistent with observed practice (respectively the entrepreneur's $\mathrm{RR}$ are $\left.1-\alpha \equiv t_{E}^{\prime}(r)\right)$. Let's take a closer look at debt. Suppose that the investor's contract is debt and the entrepreneur's contract is levered equity: $t(r)=\min \left\{r, r^{*}\right\}$ and $t_{E}(r)=\max \left\{0, r-r^{*}\right\}$. If a profit is greater than nominal debt $r^{*}$ then the property rights belong to the equityholder $\left(t_{E}^{\prime}(r)=1\right)$, and if it is less than the debt's nominal value the creditors have all property rights $\left(t^{\prime}(r)=1\right)$. This is consistent with observed practice and with theoretical literature on this topic. The same can be shown for convertible preferred participating equity and for three piece-wise securities 
like convertible preferred equity, for example.

The economic intuition behind this assumption is based on intertemporal substitution activities. Suppose an opposite situation exists where the decision-maker's first-period marginal revenue is greater than his RR. In this case, he would marginally increase the firm's current profit by decreasing the firm's second period profit, since his share in the first-period profit increase is high. Thus, the marginal revenue should serve as a good approximation for calculating residual profit rights.

\section{Conclusion}

This note has analyzed optimal financial contracts in situations where exante asymmetric information about the firm's current profit is symmetric and that about future profit is asymmetric. Since a contract on total profit is impossible to write, the standard pecking-order theory (Myers and Majluf, 1984) does not apply. A contract should then specify state-contingent current-period payoffs and residual property rights. I show that the choice of financing mode can be transformed into a stochastic bounded control problem with an isoparametric constraint (see Kamien and Schwartz, 1981). The analysis reveals that the debt is the optimal contract for the best type. This generalizes the result of Myers and Majluf (1984) and Nachman and Noe (1994) about the optimality of debt for one-period environment by taking into consideration the problem of property rights allocation in the situation where complete long term contracts are impossible.

\section{References}

[1] I. Cho and D. Kreps. Signalling games and Stable Equilibrium. Quarterly Journal of Economics. May, 102, 2, 179-121. 1987.

[2] M. Dewatripont and J. Tirole. The Theory of Debt and Equity: Diversity of Securities and Manager-Shareholder Congruence. Quarterly Journal of Economics, 109, 1027-54. 1994.

[3] M. Harris and A. Raviv. The Design of Securities. Journal of Financial Economics, 24 (2), 255-288. 1989. 
[4] O. Hart. Firms, Contracts, and Financial Structure. Oxford University Press. 1995.

[5] R. Innes. Limited Liability and Incentive Contracting with ex-ante Choices. Journal of Economic Theory, 52: 45-67. 1990.

[6] I. Jewitt. Applications of Likelihood Ratio Orderings in Economics. In Misler, K. and Scarsini, M., editors. Stochastic Orders and Decisions under Risk, volume 19. Institute of Mathematical Statistics Lecture Notes. 1991.

[7] M. Kamien and N. Scwartz. Dynamic Optimization: The Calculus of Variations and Optimal Control in Economics and Management, Series of Volumes in Dynamic Economics: Theory and applications, NorthHolland.ed. M. Wilkinson. 1981.

[8] A. Kolmogorov and S. Fomin. Introductory real analysis. Revised English Edition Translated and Edited by Richard A. Silverman. Dover publications, inc. New-York. 1970.

[9] S. Matthews. Renegotiating Moral Hazard Contracts under Limited Liability and Monotonicity. Journal of Economic Theory, 97, 1-29. 2001.

[10] F. Modigliani and M. Miller. The Cost of Capital, Corporation Finance, and the Theory of Investment. American Economic Review, 261-297. 1958 .

[11] S. Myers and N. Majluf. Corporate Financing and Investment Decisions When Firms Have Information That Investors Do not have. Journal of financial economics; 13(2), June, 187-221. 1984.

[12] D. Nachman and T. H. Noe. Optimal Design of Securities under Asymmetric information. The Review of Financial Studies Spring 1994 Vol. 7 No. 1, 1-44. 1994.

[13] E. Wolfstetter. Topics in Microeconomics. Cambridge University Press. 1999. 